\title{
Bewegung, Sport und Lernen - zwischen pädagogischem Wunsch und empirischer Wirklichkeit
}

van Ackeren, Isabell [Hrsg.]; Bremer, Helmut [Hrsg.]; Kessl, Fabian [Hrsg.]; Koller, Hans Christoph [Hrsg.]; Pfaff, Nicolle [Hrsg.]; Rotter, Caroline [Hrsg.]; Klein, Dominique [Hrsg.]; Salaschek, Ulrich [Hrsg.]: Bewegungen. Beiträge zum 26. Kongress der Deutschen Gesellschaft für Erziehungswissenschaft. Opladen; Berlin; Toronto : Verlag Barbara Budrich 2020, S. 463-475. - (Schriften der Deutschen Gesellschaft für Erziehungswissenschaft (DGfE))

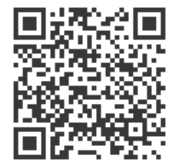

Quellenangabe/ Reference:

Gogoll, Andre; Gerlach, Erin: Bewegung, Sport und Lernen - zwischen pädagogischem Wunsch und empirischer Wirklichkeit - In: van Ackeren, Isabell [Hrsg.]; Bremer, Helmut [Hrsg.]; Kessl, Fabian [Hrsg.]; Koller, Hans Christoph [Hrsg.]; Pfaff, Nicolle [Hrsg.]; Rotter, Caroline [Hrsg.]; Klein, Dominique [Hrsg.]; Salaschek, Ulrich [Hrsg.]: Bewegungen. Beiträge zum 26. Kongress der Deutschen Gesellschaft für Erziehungswissenschaft. Opladen; Berlin; Toronto : Verlag Barbara Budrich 2020, S. 463-475 - URN: urn:nbn:de:0111-pedocs-192613 - DOI: 10.25656/01:19261

https://nbn-resolving.org/urn:nbn:de:0111-pedocs-192613

https://doi.org/10.25656/01:19261

in Kooperation mit / in cooperation with:

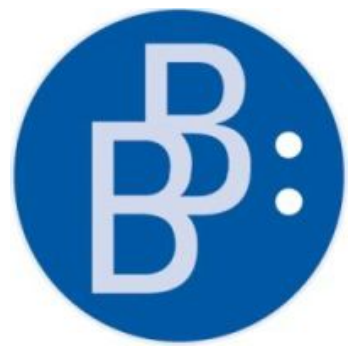

https://www.budrich.de

\section{Nutzungsbedingungen}

Dieses Dokument steht unter folgender Creative Commons-Lizenz: http://creativecommons.org/licenses/by-sa/4.0/deed.de - Sie dürfen das Werk bzw. den Inhalt vervielfältigen, verbreiten und öffentlich zugänglich machen sowie Abwandlungen und Bearbeitungen des Werkes bzw. Inhaltes anfertigen, solange sie den Namen des Autors/Rechteinhabers in der von inm festgelegten Weise nennen und die daraufhin neu entstandenen Werke bzw. Inhalte nur unter Verwendung von Lizenzbedingungen weitergeben, die mit denen dieses Lizenzvertrags identisch, vergleichbar oder kompatibel sind. Mit der Verwendung dieses Dokuments erkennen Sie die Nutzungsbedingungen an.

\section{Terms of use}

This document is published under following Creative Commons-License: http://creativecommons.org/licenses/by-sa/4.0/deed.en - You may copy, distribute and transmit, adapt or exhibit the work or its contents in public and alter, transform, or change this work as long as you attribute the work in the manner specified by the author or licensor. New resulting works or contents must be distributed pursuant to this license or an identical or comparable license.

By using this particular document, you accept the above-stated conditions of use.

\section{Kontakt / Contact:}

peDOcs

DIPF | Leibniz-Institut für Bildungsforschung und Bildungsinformation

Informationszentrum (IZ) Bildung

E-Mail: pedocs@dipf.de

Internet: www.pedocs.de

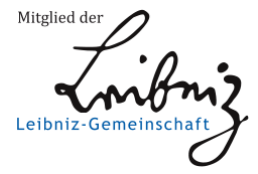




\section{Bewegungen}

Beiträge zum 26. Kongress der Deutschen Gesellschaft für Erziehungswissenschaft

Isabell van Ackeren, Helmut Bremer, Fabian Kessl, Hans Christoph Koller, Nicolle Pfaff, Caroline Rotter, Dominique Klein, Ulrich Salaschek (Hrsg.)

Schriften der Deutschen Gesellschaft für Erziehungswissenschaft

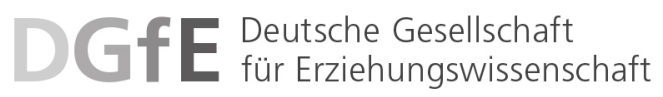


Isabell van Ackeren

Helmut Bremer

Fabian Kessl

Hans Christoph Koller

Nicolle Pfaff

Caroline Rotter

Dominique Klein

Ulrich Salaschek (Hrsg.)

\section{Bewegungen}

Beiträge zum 26. Kongress der Deutschen Gesellschaft für Erziehungswissenschaft

Verlag Barbara Budrich

Opladen • Berlin • Toronto 2020 
Bibliografische Information der Deutschen Nationalbibliothek

Die Deutsche Nationalbibliothek verzeichnet diese Publikation in der Deutschen Nationalbibliografie; detaillierte bibliografische Daten sind im Internet über

http://dnb.d-nb.de abrufbar.

(C) 2020 Dieses Werk ist bei der Verlag Barbara Budrich GmbH erschienen und steht unter der Creative Commons Lizenz Attribution-ShareAlike 4.0 International (CC BY-SA 4.0):

https://creativecommons.org/licenses/by-sa/4.0/.

Diese Lizenz erlaubt die Verbreitung, Speicherung, Vervielfältigung und Bearbeitung bei

Verwendung der gleichen CC-BY-SA 4.0-Lizenz und unter Angabe der UrheberInnen, Rechte, Änderungen und verwendeten Lizenz.

www.budrich.de

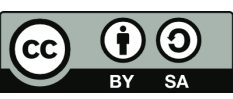

Dieses Buch steht im Open-Access-Bereich der Verlagsseite zum kostenlosen Download bereit (https://doi.org/10.3224/84742385).

Eine kostenpflichtige Druckversion (Print on Demand) kann über den Verlag bezogen werden. Die Seitenzahlen in der Druck- und Onlineversion sind identisch.

$$
\begin{array}{ll}
\text { ISBN } & 978-3-8474-2385-0 \text { (Paperback) } \\
\text { eISBN } & 978-3-8474-1553-4 \text { (PDF) } \\
\text { DOI } & 10.3224 / 84742385
\end{array}
$$

Druck: paper \& tinta, Warschau

Printed in Europe auf säurefreiem und alterungsbeständigem Papier

Umschlaggestaltung: Bettina Lehfeldt, Kleinmachnow - www.lehfeldtgraphic.de

Redaktion und Satz: Fabian Auer, Wuppertal

Typographisches Lektorat: Anja Borkam, Jena - kontakt@lektorat-borkam.de 


\section{Inhalt}

Hans-Christoph Koller

Vorwort

Fabian Kessl, Nicolle Pfaff, Isabell van Ackeren, Helmut Bremer,

Hans-Christoph Koller, Carolin Rotter, Dominique Klein, Ulrich Salaschek

Einleitung .

Käte Meyer-Drawe

Bewegungen: Viele Gemeinsamkeiten und noch mehr Unterschiede

\section{Teil I Denkbewegungen}

[Koordination: Fabian Kessl]

Christiane Thompson

„Science, not silence“. Die Öffentlichkeit der Universität an ihren Grenzen

Barbara Rendtorff, Eva Breitenbach

Frauenbewegungen, Bildung und Erziehung - Erträge und Problematiken

Britta Behm, Anne Rohstock

Loyalität. Zur verdeckten Regulierung von Denk-Bewegungen in wissenschaftlichen

Feldern. Eine Sondierung am Beispiel der Geschichte westdeutscher Bildungsforscher .... 51

Fabian Kessl

Bewegungen an den Grenzen des Disziplinären: das Beispiel von Sozialpädagogik und Sozialer Arbeit. 71

Susann Fegter, Karen Geipel, Anna Hontschik, Bettina Kleiner, Daniela Rothe, Kim-Patrick Sabla, Maxine Saborowski

Äußerungen von Sprecher*innen in einer Gruppendiskussion. Überlegungen und Analysen aus unterschiedlichen diskurs- und subjektivierungstheoretischen Perspektiven 83

Teil II Migrationsbewegungen

[Koordination: Nicolle Pfaff]

Paul Mecheril

Gibt es ein transnationales Selbstbestimmungsrecht? Bewegungsethische

Erkundungen 101 


\section{Thomas Geier}

Integration ohne Ende. Kritische Stichworte zum monothematischen Habitus der

Migrationsdebatte in Deutschland

Marcus Emmerich, Ulrike Hormel, Judith Jording, Mona Massumi

Migrationsgesellschaft im Wandel - Bildungssystem im Stillstand?

Patricia Stošić, Benjamin Rensch

„Ja, (...) wären Sie denn nicht bereit, den Lehrerberuf aufzugeben?“

Bildungsbiographische Positionierungen muslimischer Lehramtsstudentinnen im

Spannungsfeld von Pluralismusdiskurs und Diskriminierung

Arnd-Michael Nohl

Politische Erziehung. Ein blinder Fleck der Diskussion zur politischen Bildung 161

Teil III Gesellschaftliche Entwicklungen und pädagogisches Tun

[Koordination: Fabian Kessl]

Johannes Bellmann, Dirk Braun, Martina Diedrich, Katharina Maag Merki, Marcelo Parreira do Amaral, Kate Maleike

„Wer steuert die Bildung - Wer steuert die Schule?“

Ein öffentliches Podiumsgespräch zur Eröffnung des 26. Kongresses der Deutschen

Gesellschaft für Erziehungswissenschaft

Anke Wischmann, Andrea Liesner

Neu zugewanderte Jugendliche zwischen engagierter pädagogischer Hilfe,

politischen Interessen und wirtschaftlichem Kalkül

Alisha M.B. Heinemann

Learning from below - Wissen in Bewegung. $\mathrm{Zu}$ den Möglichkeiten solidarischer

Bildungsarbeit durch den 'Funds of Knowledge-Approach'

Sebastian Wachs, Wilfried Schubarth, Ludwig Bilz

Hate Speech als Schulproblem? Erziehungswissenschaftliche Perspektiven auf ein aktuelles Phänomen

Teil IV Professionalisierung in der Lehrer*innenbildung

[Koordination: Carolin Rotter]

Alexander Gröschner

Praxisbezogene Lerngelegenheiten am Beispiel lernwirksamer

Unterrichtskommunikation. „Bewegungen“ in der Aus-, Fort- und Weiterbildung

von Lehrpersonen

Julia Košinár, Anna Laros

Orientierungsrahmen im Wandel? Berufsbiographische Verläufe zwischen Studium und Berufseinstieg 
Matthias Proske, Petra Herzmann, Markus Hoffmann

Spielfilme über Lehrer/innen als Medium der kasuistischen Lehrerbildung

Kristina Geiger, Petra Strehmel

Personalentwicklung in Kindertageseinrichtungen: Maßnahmen und Strategien

von Trägern und Einrichtungen. Ergebnisse zweier empirischer Studien 283

Christina Buschle, Tina Friederich

Weiterbildung als Motor für den Erhalt von Professionalität?

Weiterbildungsmöglichkeiten für das Kita-Personal

Nikolaus Meyer, Dieter Nittel, Julia Schütz

Was haben Erzieher*innen und Professor*innen gemeinsam? Komparative

Perspektiven auf zwei stark kontrastierende pädagogische Berufsgruppen. 309

\section{Teil V Digitalisierung}

[Koordination: Isabell van Ackeren]

Manuela Pietraß

Bildung in Bewegung. Das neue Lernpotenzial digitaler Medien

Mandy Schiefner-Rohs, Sandra Hofhues, Sandra Aßmann, Taiga Brahm

Studieren im digitalen Zeitalter. Methodologische Fragen und ein empirischer Zugriff... 337

Birgit Eickelmann, Kerstin Drossel

Lehrer*innenbildung und Digitalisierung - Konzepte und Entwicklungsperspektiven .... 349

Matthias Rohs, Manuela Pietraß, Bernhard Schmidt-Hertha

Weiterbildung und Digitalisierung. Einstellungen, Herausforderungen und Potenziale ... 363

Rudolf Kammerl, Jane Müller, Claudia Lampert, Marcel Rechlitz, Katrin Potzel

Kommunikative Figurationen - ein theoretisches Konzept zur Beschreibung von

Sozialisationsprozessen und deren Wandel in mediatisierten Gesellschaften? 377

\section{Teil VI Steuerung}

[Koordination: Dominique Klein]

Michael Schemmann

„Und sie bewegt sich doch“ - Neue Steuerung und Governance in der öffentlichen

Weiterbildung.

Katharina Maag Merki

Das Educational Governance-System im Dienste der Schulentwicklung. Oder:

Wie kann Steuerung die Weiterentwicklung von Schulen unterstützen? 405 


\section{Sigrid Hartong, Annina Förschler}

Dateninfrastrukturen als zunehmend machtvolle Komponente von Educational Governance. Eine Studie zur Implementierung und Transformation staatlicher Bildungsmonitoringsysteme in Deutschland und den USA....

Tobias Feldhoff, Sabine Reh, Eckhard Klieme, Monika Mattes, Sebastian Wurster,

Brigitte Steinert, Julia Dohrmann, Christine Schmid

Schulkulturen im Wandel - Potentiale und erste Erkenntnisse zur Untersuchung von

Schulkulturen im Wandel

Felix Berth, Mariana Grgic

Wie kam die Bildung in die Krippe? Frühe Kindertagesbetreuung im Spiegel von

Wissenschaften, Recht und individuellen Einstellungen in Westdeutschland seit den 1960er-Jahren

Teil VII Körper - Leib - Bewegung

[Koordination: Fabian Kessl \& Ulrich Salaschek]

André Gogoll, Erin Gerlach

Bewegung, Sport und Lernen - zwischen pädagogischem Wunsch und empirischer

Wirklichkeit.

Maike Groen, Hannah Jäkel, Angela Tillmann, Ivo Züchner

E-Sport - Ambivalenzen und Herausforderungen eines globalen, jugendkulturellen

Phänomens.

Nino Ferrin, Benjamin Klages

Zur Kultivierung utopischer Bewegungen. Markierungen des Nicht-Verfügbaren in der Academia.

Juliane Noack Napoles

Identität als Stillstand. Ein metaphernanalytischer Blick auf eine Nicht-Bewegung.....

\section{Teil VIII Diversity / Inklusion}

[Koordination: Nicolle Pfaff]

Barbara Asbrand, Julia Gasterstädt, Anja Hackbarth, Matthias Martens

Was bewegt Inklusion? Theoretische und empirische Analysen zu

Spannungsverhältnissen einer inklusiven Schule

\section{Nina Thieme}

Zur Charakteristik der Gesellschaft, an der im Zuge von Inklusion Teilhabe ermöglicht werden soll. Vergewisserungen und Reflexionen zu möglichen Implikationen 
Bernhard Rauh, Yvonne Brandl, Michael Wininger, David Zimmermann

Inklusionspädagogik - eine halbierte Bewegung? Psychoanalytische Perspektiven

auf ein erziehungs-wissenschaftliches Paradigma

Christian Stöger

„Aber Österreich darf nicht zurückbleiben!“ Zur Wiener Hilfsschulentwicklung um 1900 . 555

Anke Karber, Gülsen Sevdiren, Kerstin Heberle, Anne Schröter, Janieta Bartz,

Tatiana Zimenkova

Hochschuldidaktische Betrachtungen differenzreflexiver Lehrer*innenbildung.

Tanja Sturm, Benjamin Wagener, Monika Wagner-Willi

Inklusion und Exklusion im Fachunterricht. Ambivalente Relationen in Schulformen

der Sekundarstufe 1

Teil IX Soziale - pädagogische Bewegungen

[Koordination: Helmut Bremer \& Jana Trumann]

Patrick Bühler

Böse Mütter im Summer of Love. Antipädagogik und Psychotherapie in den

Siebziger-Jahren

Marcel Eulenbach, Thorsten Fuchs, Yagmur Mengilli, Andreas Walther,

Christine Wiezorek

„Ich möchte Teil einer Jugendbewegung sein“"? - Jugendkultur, Protest, Partizipation.... 613

Sabrina Schenk, Britta Hoffarth, Ralf Mayer

Populismus, Protest - und politische Bildung. Soziale Bewegung(en) in

Spannungsfeldern von Affektivität, Rationalität und Praktiken der Kritik

im öffentlichen Raum

Aziz Choudry

Activist learning and knowledge production.

Autorinnen und Autoren 653 


\section{Bewegung, Sport und Lernen - zwischen pädagogischem Wunsch und empirischer Wirklichkeit}

\section{$1 \quad$ Einleitung}

An den Sport, oder allgemeiner an körperliche Bewegungen, werden eine Vielzahl pädagogischer Erwartungen herangetragen. So sollen Sport und Bewegung zunächst zu einer ganzheitlichen Entwicklung beitragen, indem sie die intellektuelle und sittliche Menschwerdung um körperliche und motorische Aspekte ergänzen. Darüber hinaus soll man in und durch Sport und Bewegung lernen können, seinen Körper ,zu bewohnen“ und ihn in gesundheitlicher Hinsicht zu achten. Sport soll weiterhin zu einer förderlichen Leistungsbereitschaft erziehen, indem man in ihm lernen kann zuzupacken, sich etwas zuzutrauen, sich Ziele zu setzen und diese konsequent und ausdauernd zu verfolgen. Ferner lernt man im Sport mit anderen zusammenzuarbeiten, sich dabei an Regeln zu halten, sich in die Perspektiven der jeweils anderen hineinzuversetzen, so dass sich positive soziale und moralische Verhaltensdispositionen, wie etwa Frustrationstoleranz oder moralische Urteilsfähigkeit, ausbilden sollten.

Viele dieser und ähnlicher Behauptungen sind inzwischen zu populären Slogans geworden und prägen oftmals unhinterfragt den pädagogischen Alltagsdiskurs über den erzieherischen oder bildnerischen Wert von Sport und Bewegung. Mittlerweile existieren aber auch eine ganze Reihe an empirischen Studien, die sich mit diesen pädagogischen Wirkungserwartungen beschäftigt haben.

Im vorliegenden Beitrag möchten wir empirische Befunde zu zumindest drei dieser Wirkungserwartungen zusammentragen. Da wir dabei in einem engen Sinne Wirkungen berichten wollen, beschränken wir uns dabei auf quantitative Studien: Im ersten Teil widmen wir uns dem populären Slogan „Toben macht schlau!“ (Zimmer 2009) und der damit verbundenen Erwartung, dass körperliche Bewegung kognitives Lernen und daraus hervorgehende akademische Leistungen fördern könne. Unter dem Titel „,(Schul-)Sport kann alles!“ geht es dann spezifischer um pädagogische Wirkungen des Sportunterrichts. Zum Schluss stellen wir unter der in Anlehnung an die Hattie-Metastudie formulierten Behauptung „Auf die Sportlehrperson kommt es an!“ aktuelle Befunde über professionelle Kompetenzen von Sportlehrpersonen dar. 
Ein Ziel unseres Beitrags ist es, an ausgewählten Beispielen aufzuzeigen, ob und inwiefern sich sport- und bewegungsbezogene pädagogische Wünsche an der empirischen Wirklichkeit messen lassen; ob wir also empirische Evidenzen haben, die die vielen und gewichtigen pädagogischen Wirkungserwartungen an Sport und Bewegung zumindest ein Stück weit erhärten können.

\section{$2 \quad$ Toben macht schlau!}

\section{$2.1 \quad$ Theoretische Grundlagen}

Die Hoffnung, die sich mit dem Slogan „Toben macht schlau!“ verbindet, ist die, dass (vor allem intensives) körperliches Bewegen Einfluss nehmen könne auf das kognitive Lernen und damit das Erbringen von intellektuellen akademischen Leistungen fördere.

Für die Schule findet man diese Argumentation heute vor allem im Konzept der „Bewegten Schule“ (z.B. Mess/Ossig/Woll 2014). Dahinter verbirgt sich ein Schulentwicklungskonzept, das seit den 1980er Jahren das Ziel verfolgt, mehr körperliche Bewegungsaktivitäten in die Schule hineinzubringen - durch bewegende Schulanlässe, auf dem Schulweg vor und nach der Schule, in den Unterrichtspausen auf dem Schulhof oder auch durch kurze, aktivierende Bewegungseinheiten im Fachunterricht selbst.

Interessanterweise wurde die Bewegte Schule in ihren Anfangsjahren vor allem mit einem gesundheitspräventiven Anspruch verknüpft: Mit dem Mehr an Bewegung wollte man ursprünglich eigentlich nur den potenziell schädlichen Einflüssen einer durch Bewegungsmangel gekennzeichneten „Sitzschule“ entgegenwirken (also etwa Haltungsschwächen ausgleichen und damit Rückenschmerzen vorbeugen). Das aktuell viel präsentere Argument der bewegungsinduzierten Lernförderung kam erst später ins Spiel - und zwar auf gleich zweifache Weise: zum einen in anthropologischen und psychomotorischen Ansätzen; zum anderen in kognitionspsychologischen und neurowissenschaftlichen Ansätzen.

1. Anthropologisch und psychomotorisch argumentierende Ansätze verstehen das körperliche Bewegen als integralen Bestandteil des kindgerechten Lernens. Sie machen den Vorteil eines bewegungsinduzierten Lernens darin aus, dass Kinder auf diese Weise quasi mit allen Sinnen lernen können - also sich Lerngegenstände nicht nur auf visuelle Weise erschließen, sondern zusätzlich auch auf eine kinästhetische Weise sprichwörtlich begreifen können. Dieser Ansatz einer bewegungsinduzierten Lernförderung in der Schule ist sicherlich der populärste Ansatz; er ist vielleicht auch der pädagogischste Ansatz, weil er nämlich die körperliche Bewegung als elementare und gleichberechtigte Form der kindlichen Weltbegegnung versteht und sie nicht als bloßes Vehikel schulischen Lernens instrumentalisiert. Leider ist er aber auch derjenige Ansatz, der (zumindest quantitativ empirisch) am schlechtesten erforscht ist, sodass wir hier von einer weiteren Berücksichtigung absehen müssen.

2. Kognitionspsychologischen und neurowissenschaftlichen Ansätzen liegt die Prämisse zu Grunde, dass körperliche Bewegungsaktivität dazu beitragen kann, solche neuronale Strukturen und neurophysiologische Prozesse zu aktivieren und zu verändern, die auch das Ausführen lernförderlicher kognitiver Prozesse begünstigen: 
- Nach der „,cognitive stimulation hypothesis“ (Tomporowski et al. 2015) geschieht das dadurch, dass bei der Bewegungsausführung zusammen mit dem motorischen Cortex zusätzlich auch solche zerebralen Areale aktiviert werden, die für kognitive Prozesse relevant sind. Dies sollte verstärkt der Fall sein, wenn die Bewegungsaktivität im Sinne einer cognitive engaging physical activity selbst mit kognitiven Aktivitäten verbunden ist, so wie das bei koordinativ anspruchsvollen Bewegungsaktivitäten der Fall ist.

- $\quad$ Eine andere Hypothese, die „cardiovascular fitness hypothesis“ (North/ McCullagh/Tran 1990), geht davon aus, dass es aufgrund von körperlicher Bewegungsaktivität zu einer Steigerung der Hirndurchblutung kommt und damit zu einer verstärkten Zufuhr von Neurotransmittern, die für die neuronale Verschaltung von Synapsen im Gehirn zuständig sind. Eine Hypothese, die vor allem greifen sollte, wenn die Bewegungsaktivitäten mit erhöhten aeroben Ausdauerbelastungen einhergehen, z.B. bei einem Dauerlauf.

Folgt man diesen Hypothesen, so tragen Bewegungsaktivitäten zu einer bewegungsinduzierten Verbesserung derjenigen kognitiven Infrastruktur bei, die für ein erfolgreicheres Lernen und bessere darauf basierende Lernleistungen erforderlich ist. Auch wenn diese Ansätze sowohl theoretisch, als auch pädagogisch und didaktisch derzeit noch viele Fragezeichen aufwerfen, so liegen hierzu bereits einige empirische Befunde vor.

\subsection{Methodische Herausforderungen}

Versucht man sich vor diesem Hintergrund einen Überblick über die aktuelle Forschungslage zum Zusammenhang von Bewegung und kognitivem Lernen zu verschaffen, dann fällt auf, wie unterschiedlich die Konstrukte sind, die zur Aufklärung dieses Zusammenhangs herbeigezogen wurden und wie unterschiedlich dementsprechend auch die Messinstrumente sind, mit denen teilweise das gleiche Konstrukt gemessen wird:

1. Auf der Seite der Kognitionen findet man häufig bereits sehr komplexe Leistungskonstrukte, wie etwa verschiedene Formen des Intelligenzkonstrukts oder die schulischen Leistungen von Schüler*innen - vor allem in Mathematik und Sprache. Häufig werden die schulischen Leistungen mit wenig validen Merkmalen gemessen, so etwa mit der Fachnote oder über Leistungseinschätzungen der Lehrpersonen oder von Mitschüler*innen. Zuweilen kommen aber auch standardisierte Testverfahren zum Einsatz, die jedoch nur einzelne Leistungsbereiche des Faches in den Vordergrund stellen. Auf einer mittleren Komplexitätsstufe finden wir solche Konstrukte, die einzelne kognitive Leistungsbereiche repräsentieren sollen, wie etwa Sprachfähigkeit, Wahrnehmungsleistungen, Konzentrationsleistungen, Memorierungsleistungen, schlussfolgerndes Denken oder Kreativität. Schließlich auf fundamentalster Stufe werden einzelne generische kognitive Prozesse herangezogen, die den komplexeren kognitiven Leistungen zu Grunde liegen. Dazu gehören etwa die optische Differenzierungsfähigkeit, die eine wesentliche Voraussetzung für Intelligenzleistungen darstellt, oder die sog. Exekutiven Funktionen (Updating, Inhibition und Shifting), die eigentlich allen anderen höheren kognitiven Prozessen und unserer Fähigkeit zu einem endogen kontrollierten, selbstgesteuerten, zielgerichteten Verhalten $\mathrm{zu}$ Grunde liegen.

2. Auf der Seite der Bewegung sind für die Analyse von Zusammenhängen zwischen Bewegungsaktivitäten und kognitivem Lernen ebenfalls eine Vielzahl sehr unterschiedlicher Merkmale herangezogen worden. Zunächst fallen eine Reihe an Studien auf, in denen gar keine Bewegungsaktivitäten berücksichtigt werden, sondern vielmehr deren Ergebnisse, wie etwa körperliche Fitnessmaße. Studien dagegen, in denen die Bewegungen der untersuchten Kinder direkt 
eingeflossen sind, weisen eine breite Palette an Operationalisierungsvarianten für die berücksichtigten Aktivitäten auf. Sie lassen sich erstens nach ihrer Art unterscheiden, also etwa danach, ob alltägliche Bewegungsaktivitäten von Kindern berücksichtigt wurden oder ob ein spezifisches Bewegungsprogramm durchgeführt wurde. Für die spezifischen Bewegungsprogramme lässt sich weiter ausdifferenzieren, ob sie eigens angereichert wurden mit besonderen kognitiven Herausforderungen (cognitive engaging physical activity). Die Aktivitäten lassen sich zweitens nach ihren Inhalten unterscheiden. Häufig untersucht wurden Ausdauer-, Kraftoder Koordinationstrainings; aber etwa auch Entspannungsübungen oder bewegungsaktive Spiele. Drittens finden sich zeitlich und dynamisch unterschiedliche Varianten, so etwa nach der Regelmäßigkeit der eingeflossenen Bewegungsaktivitäten - (längerfristige ,,chronische Aktivitäten“" vs. kurzfristige ,,akute Aktivitäten“), nach der Dauer und Intensität der durchgeführten Bewegungseinheit. Viertens schließlich unterscheiden sich die berücksichtigten Bewegungsaktivitäten nach ihren kontextuellen Bedingungen: Erfolgte das Training durch einzelne Übungen oder wurden dazu Spiele gemacht? In welchem Setting fanden die Bewegungsaktivitäten statt? Wie wurden die Aktivitäten organisiert? Wie groß waren die Gruppen? Wer leitete das Bewegungsprogramm an?

Insgesamt zeigt sich ein sehr differenziertes Bild der aktuellen Forschung zum Zusammenhang von Bewegung und kognitivem Lernen: eine ungeheure Vielfalt an kognitiven Konstrukten, eine ungeheure Vielfalt an körperlichen Aktivitäten und - über die dargestellten Merkmale hinaus - eine ungeheure Vielfalt an Forschungsdesigns (viele Querschnittsstudien, einige Längsschnittstudien, in jüngerer Zeit vermehrt Interventionsstudien) und auch eine Vielfalt an Stichproben, die sich z.T. sehr stark unterscheiden.

\section{$2.3 \quad$ Empirische Befunde}

Das alles spricht eigentlich gegen eine globale Einschätzung der bisher erarbeiteten Befunde. Dennoch existieren eine ganze Reihe an Meta-Analysen, die insgesamt zu leicht positiven, kleinen Effektgrößen kommen, von Hedge's g = 0.25 über alle Studien bis 1997 (Etnier et al. 1997) und $g=0.32$ bis 2003 und speziell für die Studien, die mit Kindern und Jugendlichen durchgeführt wurden (Sibley/Etnier 2003). Größer ist der Effekt, wenn die Bewegungsaktivitäten nicht länger als 15-20 Minuten andauern und mit einer Intensität von 65\% durchgeführt werden würden.

Einen deutlich spezifischeren Blick auf die Zusammenhänge zwischen Bewegung und Lernen eröffnen Studien, die sich mit dem Einfluss von Bewegung, Spiel und Sport auf die exekutiven Funktionen von Kindern und Jugendlichen beschäftigen. Exekutive Funktionen sind elementare kognitive Prozesse, die u.a. dafür sorgen, dass relevante Informationen eine kurze Zeit lang im Arbeitsgedächtnis präsent gehalten und aktiv weiterbearbeitet werden können. Die Fähigkeit zu einem solchen Updating ermöglicht es uns etwa, Alternativen abzuwägen, um in konkreten Situationen angemessen handeln zu können. Exekutive Funktionen sind darüber hinaus dafür verantwortlich, dass wir Störreize ausblenden können (Inhibition) oder dass wir unseren Aufmerksamkeitsfokus flexibel verlagern können, wenn sich die Relevanz von Reizen plötzlich verändert (Shifting).

Eine erste Studie, die hier exemplarisch vorgestellt werden soll, weist den exekutiven Funktionen eine Mediatorenrolle zwischen Bewegungsaktivitäten und schulischen Lernleistungen zu (Schmidt et al. 2017). 
Untersucht wurden die motorischen Fähigkeiten (Ausdauer, Kraft und Schnelligkeit), die exekutiven Funktionen und die schulischen Leistungen in Mathematik, Lesen und Schreiben von 236 Kindern im Alter von 10-12 Jahren (53.5\% Mädchen, $\mathrm{M}=11.3$ Jahre, $\mathrm{SD}=.62$ ) über drei Messzeitpunkte. Der Zusammenhang von motorischen Fähigkeiten und den Schulleistungen der untersuchten Schüler*innen beträgt $\beta=.47$. Werden die exekutiven Funktionen als Mediator zwischengeschaltet, so sinkt der direkte Zusammenhang und wird auch nicht-signifikant. Im Gegenzug zeigt sich nun ein signifikanter, über die exekutiven Funktionen vermittelter Einfluss von insgesamt $\beta=.32$ (s. Abbildung1). Allerdings zeigt sich in der Studie auch, dass dieser Mediatoreffekt ausschließlich von den koordinativen Fähigkeiten der untersuchten Kinder ausgeht. Andere motorische Fähigkeiten (Ausdauer, Kraft) weisen keine signifikante direkte Beziehung zu den exekutiven Funktionen auf. Insgesamt gibt die berichtete Studie erste Hinweise darauf, dass exekutive Funktionen vor allem durch solche Aktivitäten gefördert werden können, deren Ausführung von den Schüler*innen zusätzlich zu den körperlich-motorischen auch exekutiv kontrollierte Leistungen abverlangen - wie dies bei einem Koordinationstraining der Fall ist.

Abbildung 1: Mediatorenrolle der exekutiven Funktionen zwischen motorischen Fähigkeiten und schulischen Lernleistungen

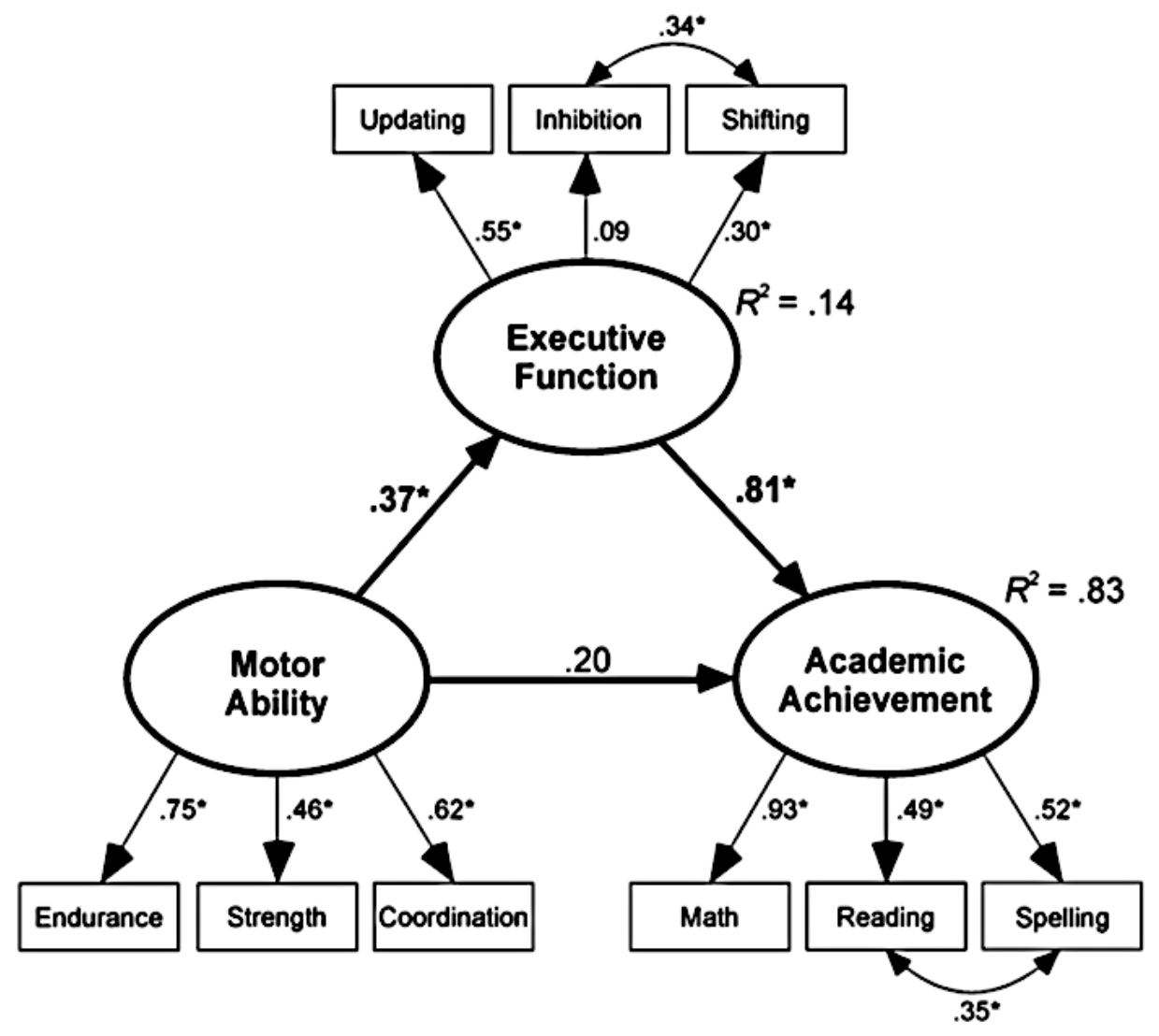

Quelle: Schmidt et al. 2017 
Dieser Spur sind auch zwei weitere Interventionsstudien zum Einfluss von Bewegungsaktivitäten auf die exekutiven Funktionen gefolgt. Bei ihnen kommt hinzu, dass sie mit Bewegungsaktivitäten gearbeitet haben, die einen explizit kognitiv-exekutiven Anspruch enthalten und sowohl fachdidaktische als auch adressatenspezifische Überlegungen einbezogen haben.

Eckenbach (2017) hat für ihre Studie eigene Sport- und Bewegungsspiele entwickelt und getestet, die nicht nur bewegungsbasiert sind und auf eine Förderung von exekutiven Funktionen zielen, sondern gleichzeitig auch fachdidaktischen Ansprüchen genügen und sich somit in den alltäglichen Sportunterricht integrieren lassen. Ein Beispiel für ein solches Spiel ist Catch or not; eine Abwandlung des den meisten Schüler*innen bekannten Spiels Ball über die Schnur. Gegeneinander spielen zwei Mannschaften, die einen Spielball so über eine gespannte Schnur oder über ein Netz spielen müssen, dass er von der gegnerischen Mannschaft nicht erreicht werden kann. Begonnen wird mit einem Softball, der von den Spieler*innen gefangen werden muss, ohne, dass er den Boden berührt. Nach einer Weile kommt zusätzlich ein Volleyball hinzu, für den nun auch noch andere Regeln gelten - so etwa die, dass der Ball erst einmal auf den Boden auftrumpfen muss, bevor man ihn fangen darf. In der für die Inhibitionsleistung anspruchsvollsten Form wird das parallele Spielen von zwei Bällen um einen zusätzlichen Luftballon, der die ganze Zeit über in der Luft gehalten werden muss, ergänzt.

Stichprobe in dieser Interventionsstudie waren 197 Kinder (51\% Mädchen, M = 11.96 Jahre, SD $=.46$ ) von denen 97 Schüler*innen ganz normal am Sportunterricht teilgenommen haben (Kontrollgruppe). Bei den 100 Kindern aus der Experimentalgruppe wurde der Sportunterricht über 20 Wochen lang (je 30 Minuten pro Woche) angereichert um eines der erarbeiteten Bewegungsspiele. Vor und nach der Intervention wurden die exekutiven Funktionen getestet. Insgesamt zeigte sich, dass die entwickelten Spiele insbesondere die mit dem Stroop-Test getestete Inhibitionsfähigkeit der Schüler*innen beeinflussen, also ihre Fähigkeit Störreize auszublenden. So ergab sich ein kleiner Effekt (eta $\left.{ }^{2}=0.026\right)$, der noch etwas größer ausfällt, wenn man nur diejenigen Gruppen einbezieht, bei denen die Intervention nach Wahrnehmung der Kinder gut umgesetzt wurde. Am größten ist der Effekt, wenn man nur diejenigen Kinder in die Auswertung einbezieht, die in ihrer Inhibitionsleistung zum Pre-Test zu den $20 \%$ der schlechtesten gehörten. Diese Gruppe, die zu Beginn der Studie Störreize am schlechtesten ausblenden konnte, profitiert demnach am meisten von den durchgeführten Bewegungsspielen.

Dieser besonderen Personengruppe widmen sich Benzing, Chang und Schmidt (2018), die ihre Interventionsstudie ausschließlich mit Kindern mit einem diagnostizierten ADHS durchgeführt haben. Da es sich dabei um eine Population handelt, die Schwierigkeiten hat, normalen, regelmäßigen Sportangeboten nachzugehen, geschweige denn längerfristig ein kontrolliertes Bewegungsprogramm durchzuhalten, wurde in dieser Studie eine besondere Form von Bewegungsaktivitäten berücksichtigt, die für die Kinder mit ADHS fesselnder sein sollten, nämlich ein Exergaming-Fitnessspiel. Exergames sind Computerspiele, in denen man sich durch körperliche Bewegungen vor einer Kamera als Spielfigur im medialen Spielgeschehen selbst steuern kann. Derartige Spiele stellen dabei ein hohes Maß an körperlichen und kognitiven Anforderungen, sollten aber dennoch Kinder mit ADHS zum längeren Durchhalten motivieren.

An der Studie teilgenommen haben 22 Kinder in der Experimentalgruppe $(\mathrm{M}=10.45$ Jahre, SD $=1.34)$ und 22 Kinder in der Kontrollgruppe $(\mathrm{M}=10.32, \mathrm{SD}=1.46)$. Die Kinder der Experimentalgruppe haben acht Wochen lang mindestens 30 Minuten pro Woche ein Exergame-Fitnessspiel 
durchgeführt. Erste Ergebnis der zum aktuellen Zeitpunkt noch nicht abgeschlossenen Studie bestätigen den insgesamt positiven Einfluss kognitiv anspruchsvoller Bewegungsaktivitäten auf das Shifting, also der exekutiven Funktion, die für die Fokussierung der Aufmerksamkeit verantwortlich ist. $\mathrm{Ob}$ die in der Studie genutzten besonderen Bewegungsaktivitäten über die Verbesserung der exekutiven Funktionen vermittelt auch einen Beitrag zur Verminderung der ADHS-Symptome bei den Kindern leisten können, wird derzeit noch untersucht.

Insgesamt zeigen die bisher erarbeiteten Befunde, dass körperliche Bewegungen - insbesondere dann, wenn sie mit erhöhten kognitiven Anforderungen einhergehen - zumindest elementare kognitive Prozesse positiv beeinflussen können. Es wird noch zu zeigen sein, ob sich mit den gesteigerten Aufmerksamkeitsleistungen auch das Lernverhalten von Kindern verbessert und das Toben Schüler*innen letztendlich nicht nur aufmerksamer, sondern auch schlauer werden lässt.

\section{$3 \quad$ Schulsport kann alles!?}

Nach dem fokussierten Blick der Wirkungen von Bewegungsaktivitäten auf die Kognition wollen wir nun den Fokus auf das Fach Sport legen. Die Palette an Wirkungshoffnungen für den Sport im Allgemeinen (vgl. Gerlach/Brettschneider 2013) ist bereits eingangs geschildert worden. Diese Hoffnungen werden oft für den Sportunterricht in der Schule übertragen, ohne dass immer das Setting, die Struktur und die Bedingungen, unter denen Sport und Bewegung stattfinden, angemessen berücksichtigt werden. In diesem Zusammenhang werden Effekte in verschiedensten Dimensionen diskutiert, die zuweilen zum Eindruck verleiten könnten, dass der Schulsport einfach alles kann. Wahrscheinlich wird an kaum ein Fach ein derartiges Spektrum an Wirkungshoffnungen herangetragen, die einem Breitbandantibiotikum ähneln. $\mathrm{Zu}$ den verschiedenen Outputmerkmale gehören folgenden Bereiche:

- Motorische Outputmerkmale (z.B. motorische Basiskompetenzen, motorische Fertigkeiten und Fähigkeiten)

- Kognitive Outputmerkmale (z.B. Reflexions- und Urteilsfähigkeit im und über Bewegung, Spiel und Sport, kognitive Leistungsfähigkeit)

- Dispositionale Outputmerkmale (z.B. fachspezifisches Interesse, Anstrengungsbereitschaft, fachliches Selbstkonzept)

- $\quad$ Emotionale Outputmerkmale (z.B. Freude und Spaß, verminderte Angst, Stressreduktion)

- $\quad$ Soziale Outputmerkmale (z.B. Empathie, Fairness, soziales Verhalten)

- Behaviorale Outputmerkmale (z.B. Teilhabe am außerschulischen Sport, körperliche Aktivität, aktiver Lebensstil)

- $\quad$ Gesundheitliche Effekte (Fitness, physische, psychische und soziale Ressourcen)

Potenzielle Wirkungen des Sportunterrichts werden in der Schuleffektivitätsforschung i.d.R. im Angebot-Nutzen-Modell diskutiert (Fend 1981, Helmke 2013, Kunter/Trautwein 2013). Seiler (2018) hat für das Fach Sport eine wichtige Unterscheidung bei den Wirkungen vorgenommen, in dem sie kurzfristige (Output), mittelfristige (Outcome) und langfristige Effekte (Impact) unterscheidet. Beispielsweise sollte ein Sportunterricht kurzfristig zu einer Förderung von motorischen Kompetenzen und sportaffinen Dispositionen führen, die Schü- 
ler*innen mittelfristig dazu befähigen, an der Bewegungs-, Spiel- und Sportkultur teilzuhaben. Langfristig sollte dies u.a. zu einem erhöhten Fitnesszustand führen, der als wichtige Ressource im Prozess der Aufrechterhaltung der Gesundheit dient.

Insgesamt ist der Forschungsstand einer Effektivitätsforschung im Sportunterricht noch sehr überschaubar, da nur wenige Studien existieren, die mehrere Aspekte innerhalb des Angebot-Nutzen-Modells in einen direkten Zusammenhang stellen und längsschnittlich mit Blick auf Wirkungen analysieren. Hierzu zählt die Basler Wirkungsstudie IMPEQT, ${ }^{1}$ in der von ca. 1.100 Jugendlichen und 48 Sportlehrpersonen aus der Sekundarschule über ein Jahr hinweg Merkmale der Lehrpersonen, Prozessmerkmale guten Unterrichts und Outputmerkmale erhoben wurden. Im Folgenden sollen zentrale Ergebnisse zur Wirkung der Unterrichtsqualität auf Ergebnismerkmale berichtet werden.

Das Thema „Unterrichtsqualität“ ist auch im Fach Sport ein Forschungsfeld, das zunehmend bearbeitet wird (Herrmann/Gerlach 2019). Dis Diskussion dreht sich u.a. um die Frage, ob Aspekte der Unterrichtsqualität generisch oder fachspezifisch sind. Herrmann, Pühse, Seiler und Gerlach (2015) unternahmen eine erste empirische Annäherung an das Thema, indem sie einzelne Merkmale guten Unterrichts vor dem Hintergrund der drei Basisdimensionen guten Unterrichts (1) Klassenführung, (2) Unterrichtsklima und (3) Aktivierung modellierten. Bei der Prüfung zeigte sich, dass sich die einzelnen Merkmale in zwei übergeordneten Basisdimensionen bündelten. Die Dimension Klassenführung wurde durch die Merkmale Disziplin, Zielklarheit, Regelklarheit und Diagnosekompetenz abgebildet, während die Dimension schüler*innenorientiertes Unterrichtsklima durch die Merkmale Fürsorglichkeit, Mitbestimmung der Schüler*innen, individuelle Bezugsnormorientierung und Differenzierung repräsentiert wurden. Auf die Erfassung der Basisdimension (kognitive) Aktivierung wurde in dieser Studie verzichtet, da zunächst auf einer theoretischen Ebene eine fachspezifische Adaptation notwendig erschien, bevor eine Operationalisierung und Erhebung möglich wäre (vgl. hierzu Niederkofler/Amesberger 2016, Wibowo et al. 2019). Insgesamt konnte gezeigt werden, dass die einzelnen Merkmale im Fach Sport eine ähnliche Strukturierung aufwiesen, wie dies auch in anderen Fächern der Fall ist.

Diese Qualitätsdimensionen und -merkmale wurden in der Folge u.a. in ihrem Einfluss auf Interesse, Anstrengungsbereitschaft und Selbstkonzept analysiert (Seiler 2019, Herrmann/Gerlach in Überarb.). Dabei zeigte sich, dass der Einfluss der Klassenführung auf die Veränderung der motivationalen und volitionalen Merkmale deutlich größer ist als der des schüler*innenorientierten Unterrichtsklimas, für das ursprünglich ein größerer Einfluss vermutet wurde. Der Befund verweist auf die besondere Bedeutung des Klassenmanagements für alle Lehr- und Lernprozesse im Fach, was sich gut durch die spezifischen Rahmenbedingungen begründen lassen kann (Sport als „Handlungsfach“, räumliche Besonderheiten, ständiger Fluss im Unterricht, körperliche Exponiertheit, kompetitive Elemente und Sicherheitsaspekte), und in Forschung und Sportlehrerbildung eine besondere Aufmerksamkeit verdient (Linka/Gerlach 2019).

1 „Implementation in Physical Education and the Quality of Teaching“ gefördert von der Eidgenössischen Sportkommission (ESK) 2011-2013 (Leitung: Erin Gerlach) 


\section{$4 \quad$ Auf die Lehrperson kommt es an!}

Nicht erst seit den Ergebnissen der Hattie-Studie (Hattie 2009) wird den professionellen Voraussetzungen von Sportlehrer*innen eine hohe Bedeutung für die Unterrichtsqualität und den Lernerfolg von Schüler*innen im Fach Sport zugeschrieben. Theoretisch folgen auch diese Arbeiten der Annahme, dass professionelle Handlungskompetenzen das professionelle Handeln von Sportlehrpersonen bei der Planung und bei der Durchführung von Sportunterricht beeinflussen und vermittelt über die Unterrichtsqualität für die Lernerfolge verantwortlich sind (z.B. Baumert/Kunter 2006)

Unser Überblick über die aktuelle Forschungslage zu professionellen Handlungskompetenzen von Sportlehrer*innen fokussiert ihr professionelles Wissen und ihre motivationalen Orientierungen.

\subsection{Professionelles Wissen von Sportlehrpersonen}

Verschafft man sich zunächst einen Überblick über die Studien zum Professionswissen von Sportlehrpersonen, fällt auf, dass die für bedeutsam erachteten Wissensfacetten auf sehr unterschiedliche Weise operationalisiert werden (vgl. Vogler et al. 2018).

Das dürfte zum einen damit zusammenhängen, dass es dazu auch in den in der Sportdidaktik rezipierten großen Studien zu professionellen Kompetenzen von Lehrpersonen, wie etwa die COACTIV-Studien oder die TEDS-M-Studien, sehr unterschiedliche Vorstellungen gibt. Zum anderen dürfte diese Uneinigkeit auch darin begründet sein, dass sich die fachbezogene Forschung, ebenso wie die Schulpraxis, durch eine große Vielfalt an Fachkonzeptionen auszeichnen. Sie reichen von der Vorstellung, der Sportunterricht sei vor allem zum Schwitzen da, bis hin zu der Idee, dass das Fach sein Bildungspotenzial vornehmlich in der Vermittlung einer ästhetisch-expressiven Weltbegegnung entfalte. Je nachdem, welcher Fachkonzeption man folgt, kommt man zu unterschiedlichen Vorstellungen davon, was nun das für das professionelle Handeln relevante Wissen sei. Insgesamt ist aufgrund dieser Vielfältigkeit an Modellierungen und Operationalisierungen die unmittelbare Vergleichbarkeit der erzielten Befunde erheblich einschränkt.

Eine der profiliertesten Studie zum professionellen Wissen stammt von Brühwiler und Kollegen (2017). Bei ihrer Modellierung folgen sie dem heuristischen Modell der TEDS-MStudie und separieren bei der Testung zunächst das Fachwissen und fachdidaktisches Wissen. Beide Wissensdimensionen werden auf einen spezifischen Gegenstandsbereich des Sportunterrichts bezogen (Bewegen an Geräten - Rollen und Drehen): Das gegenstandsspezifische Fachwissen wird unterteilt in sportartenspezifisches Wissen und bewegungswissenschaftliches Wissen (z.B. biomechanische Prinzipien); das fachdidaktische Wissen wird bezogen auf die Planung, Durchführung und Auswertung von Unterricht. Das kognitive Niveau des Wissens wird analog der TEDS-M-Studien unterteilt in Wissen und Kennen (z.B. Kenntnis über verschiedene Hilfestellungen zum Erlernen einer Laufkippe am Reck), Wissen und Anwenden (z.B. aufzeigen, wie man diese Lernunterstützung am besten anwendet) und Urteilen und Begründen (z.B. eine Hilfestellung theoriegeleitet begründen können).

Insgesamt konnten nach der Pilotierung je 19 Items für die Testung des Fachwissens und des fachdidaktischen Wissens von Sportlehrpersonen genutzt werden. Untersucht wurden 219 Versuchspersonen - sowohl Lehrpersonen der Primarstufe und der Sekundarstufe I als 
auch Studierende an der Pädagogischen Hochschule St. Gallen. Erfasst wurden neben dem professionellen Wissen noch weitere Merkmale der Professionskompetenz, u.a. die Überzeugungen der Sportlehrperson im Hinblick auf das richtige Lehren und Lernen im Sport - also sind Lehren und Lernen eher transmissionsorientiert zu denken oder wird der Lehr-Lernprozess eher als wechselseitiger Ko-Konstruktionsprozess verstanden. Als Kriterium wurden die Planungsüberlegungen der Sportlehrpersonen erfasst. Hierzu wurde den Sportlehrpersonen eine standardisierte Planungsaufgabe erteilt und ein Planungsraster vorgegeben, in dem sie ihre Eintragungen machen konnten. Diese Eintragungen wurden dann nach fünf Kriterien im Hinblick auf den Grad ihrer Richtigkeit bewertet.

Skaliert wurden die Testdaten zum Professionswissen mit Hilfe des Rasch-Modells (Abbildung 1). Die Analyse der Verteilung der Itemschwierigkeiten macht darauf aufmerksam, dass viele Aufgaben entweder zu schwer oder zu leicht für die untersuchten Sportlehrpersonen waren. Darüber hinaus zeigte sich für die Passung zwischen Itemschwierigkeiten und Personenfähigkeiten, dass die Personenfähigkeiten eher im unteren Bereich liegen. Abbildung 2 bildet die Ergebnisse der Modellprüfung ab. Es zeigt sich, dass das 2-Faktoren-Modell mit den beiden Wissensdimensionen Fachwissen und fachdidaktisches Wissen, die Daten signifikant besser abbilden kann, als das 1-Faktorenmodell. Darüber hinaus deutet die latente Korrelation zwischen den beiden Wissensdimensionen von $r=.72$ darauf hin, dass es sich bei dem getesteten Fachwissen und fachdidaktischen Wissen zum Rollen und Drehen an Geräten eher um zwei unterscheidbare Konstrukte handelt.

Abbildung 2: 2-Faktormodell für das fachliche und fachdidaktische Wissen auf Basis der Summenscores der Unterdimensionen (geparcelt; Korrelation auf latenter Ebene; standardisierte Schätzwerte)

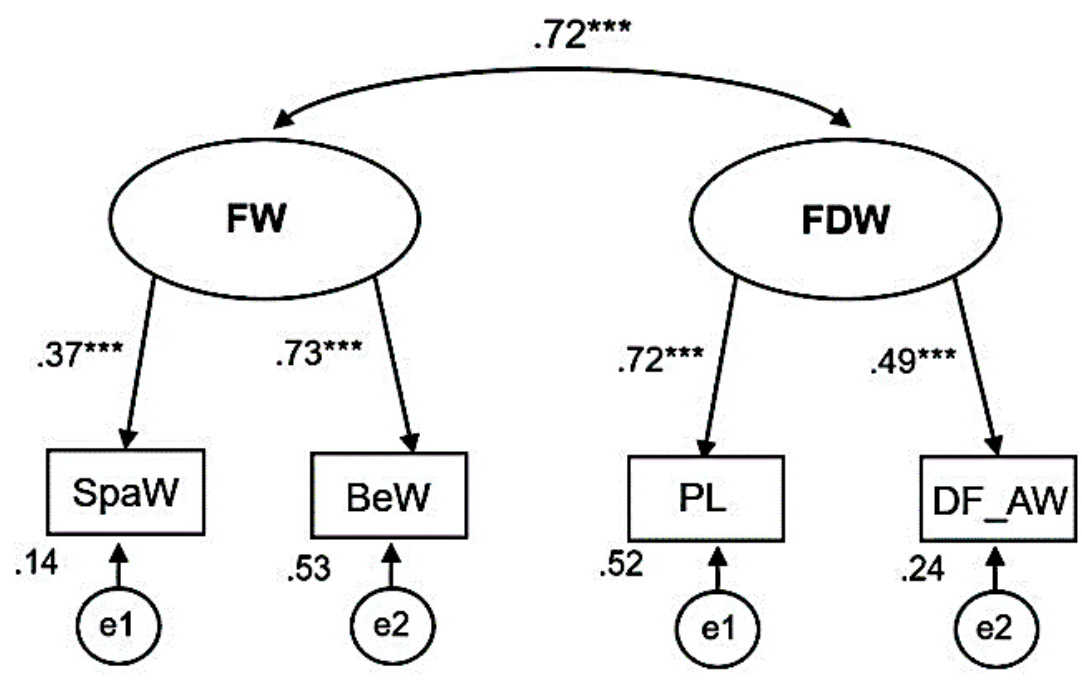

Quelle: Brühwiler et al. 2018 
Als Ergebnis der Testung des professionellen Wissens zeigt sich erstens, dass sich die bereits unterrichtenden Sportlehrpersonen in ihren Wissenswerten nur wenig von den Studierenden unterscheiden. So haben die unterrichtenden Lehrpersonen tendenziell ein höheres Fachwissen; die Studierenden tendenziell ein marginal höheres fachdidaktisches Wissen in Bezug auf das Rollen und Drehen an Geräten. Die einzig bedeutsameren Unterschiede im professionellen Wissen zeigen sich in der Subgruppe der Studierenden - und zwar in der Weise, dass die Studierenden der Sekundarstufe I sowohl im Fachwissen, als auch in ihrem fachdidaktischen Wissen den angehenden Primarlehrpersonen überlegen sind. Hinter diesem Befund dürften die unterschiedlichen Studienanteile der Zielstufen stehen. So haben angehende Primarlehrpersonen als Generalisten im Unterschied zu den Sek-I-Studierenden lediglich einen sehr geringen Anteil an sportdidaktischer Ausbildung in ihrem Studium.

Zweitens zeigen die Ergebnisse, dass das professionelle Handeln der untersuchten Personen - gemessen an ihren Planungsüberlegungen - völlig unabhängig zu sein scheint, von ihrem professionellen Wissen: weder das fachdidaktische Wissen, noch das Fachwissen weisen bedeutsame Zusammenhänge mit der Qualität der Planung auf. Was sich dagegen erwartungskonform zeigt, sind die Zusammenhänge zwischen den professionellen Überzeugungen der Sportlehrpersonen und ihren Planungsüberlegungen: je größer die Transmissionsorientierung in den Lehr-Lernüberzeugungen, desto dürftiger sind die Planungsüberlegungen; je stärker dagegen die Überzeugung, Lehren und Lernen im Sportunterricht sei eine wechselseitige Ko-Konstruktion, desto zutreffender waren auch die Überlegungen zur Planung.

\subsection{Motivationale Orientierungen von Sportlehrpersonen}

Wie sieht es nun mit den fachbezogenen motivationalen Orientierungen der Sportlehrpersonen aus? In welchem Zusammenhang stehen intrinsisch-motivationale und affektive Merkmale wie Interesse am Sport und Sportunterricht, intrinsische Selbstregulation oder fachlicher Enthusiasmus zum professionellen Handeln im Sportunterricht? Wie werden Prozessmerkmale des Sportunterrichts, wie etwa die Schüler*innenorientierung, Kompetenzunterstützung oder die Schaffung eines lernförderlichen Klimas durch die fachbezogene motivationale Orientierung der Sportlehrpersonen beeinflusst?

In der hierzu berichteten Studie von Büchel (2019) wurden insgesamt N = 106 Sport unterrichtende Lehrpersonen zu einer breiten Palette an sport- und sportunterrichtsbezogenen motivationalen Orientierungen (also einmal zum Fach Sport als solches und einmal zum Unterrichten im Fach Sport) befragt. Zusätzlich wurden die Schüler*innen dieser Lehrpersonen befragt und zwar dazu, wie sie das Verhalten ihrer Lehrer*innen in Bezug auf die Unterrichtsqualität wahrnehmen - also in Bezug auf die Schüler*innenorientierung der Lehrperson, ihre Kompetenzunterstützung, ihr Achten auf Sicherheit, ihr Bemühen um ein lernförderliches Klima, ihre Klassenführung und ihre Zielorientierung.

Analysiert wurden die erhobenen Daten auf Klassenebene mit Mehrebenenregressionsmodellen, wobei die Unterrichtswahrnehmung der Schüler*innen als abhängige Variable eingegangen ist, die einzelnen motivationalen Orientierungen der Lehrpersonen als unabhängige Variablen. Zusätzlich aufgenommen wurden zwei latente Konstrukte 2. Ordnung: zum einen die motivationalen Orientierungen der Lehrpersonen, die sich auf das Fach Sport als Gegenstand richten; zum anderen diejenigen motivationalen Orientierungen, die sich auf das Unterrichten von Sport als Tätigkeit beziehen. 
Im Ergebnis zeigt sich, dass Schüler*innen verschiedene Prozessmerkmale des Sportunterrichts bei Lehrpersonen mit einer hohen intrinsischen und interessegeleiteten Motivation, mit einem großen Enthusiasmus für Fach und Unterricht und mit positiveren Emotionen grundsätzlich als besser beurteilen als bei Lehrpersonen, bei denen die motivational-affektive Ausgangslage nicht so günstig ausgeprägt ist. Dies gilt in besonderem Maße für die Einschätzungen zum lernförderlichen Klima und zur Kompetenzunterstützung, also zu solchen Prozessmerkmalen, bei denen es eher um die Schaffung einer motivational-emotionalen Lernbereitschaft bei den Schüler*innen geht. Der Effekt zeigte sich dagegen nicht für solche Unterrichtsmerkmale, die nach Helmke das Lernen der Schüler*innen auf direkte Weise fördern sollen, wie etwa die Klärung von Lernzielen oder die Optimierung der Lernzeit durch ein gutes Klassenmanagement. Hier gibt es gar keine signifikanten Bezüge zu den motivationalen Orientierungen der Sportlehrpersonen.

\section{$5 \quad$ Schluss}

In unserem Beitrag haben wir Befunde zusammengetragen, die die hohen pädagogischen Erwartungen, die an den Sport und an Bewegung herangetragen werden, mit empirischer Evidenz konfrontieren. Dabei sollte zum einen deutlich werden, dass inzwischen eine beachtliche Anzahl an Studien existieren, die sich um eine theoretische und empirische Aufklärung dieser teilweise sehr plakativen Behauptungen bemühen. Zum anderen sollte aber auch deutlich geworden sein, dass diese Studien in ihrem konzeptionellen und methodischen Vorgehen noch sehr disparat sind und bedingt zu belastbaren Ergebnissen geführt haben. Es würde uns sehr freuen, wenn wir mit unserem Beitrag zur weiteren Mitarbeit bei der Erforschung der Zusammenhänge zwischen Bewegung, Sport und Lernen motivieren konnten.

\section{Literatur}

Benzing, Valentin/Chang, Yu-Kai/Schmidt, Mirko (2018): Acute Physical Activity Enhances Executive Functions in Children with ADHD. In: Scientific reports, 8, 1, p. 12382.

Brühwiler, Christian/Büchel, Sonja/Egger, Patrick/Hochweber, Ann Christin/Kolovou, Dimitra/Perret, Jacqueline (2018): Professionelle Kompetenzen sportunterrichtender Lehrpersonen. Schlussbericht zum Forschungsprojekt. St. Gallen: Pädagogische Hochschule.

Baumert, Jürgen/Kunter, Mareike (2006): Stichwort: Professionelle Kompetenz von Lehrkräften. In: Zeitschrift für Erziehungswissenschaft, 9, 4, S. 469-520.

Büchel, Sonja (2019): Lehrermotivation im Sportunterricht. Wiesbaden: Springer VS.

Eckenbach, Karin (2017): Games for Brains. Spielerische Lernförderung durch Bewegung. Seelze: Klett/Kallmeyer.

Etnier, Jennifer L./Salazar, Walter/Landers, Daniel M./Petruzzello, Steven J./Han, Myungwoo/Nowell, Priscilla (1997): The influence of physical fitness and exercise upon cognitive functioning: A metaanalysis. In: Journal of Sport and Exercise Psychology, 19, 3, pp. 249-277.

Fend, Helmut (1981). Theorie der Schule. München: Urban \& Schwarzenberg (2. durchgeseh. Aufl.).

Gerlach, Erin/Brettschneider, Wolf-Dietrich (2013): Aufwachsen mit Sport. Befunde einer 10-jährigen Längsschnittstudie zwischen Kindheit und Adoleszenz. Aachen: Meyer \& Meyer. 
Hattie, John A.C. (2009): Visible learning: A synthesis of over 800 meta-analyses relating to achievement. London: Routledge.

Helmke, Andreas (2013). Unterrichtsqualität und Lehrerprofessionalität. Seelze-Velber: Friedrich (5. überarb. Aufl.).

Herrmann, Christian/Seiler, Sara/Pühse, Uwe/Gerlach, Erin (2015): "Wie misst man guten Sportunterricht?" - Erfassung zentraler Dimensionen von Unterrichtsqualität im Schulfach Sport. In: Zeitschrift für sportpädagogische Forschung, 3, 1, S. 5-26.

Herrmann, Christian/Gerlach, Erin (2019): Unterrichtsqualität im Fach Sport - Ein Überblicksbeitrag zum Forschungsstand in Theorie und Empirie. Manuskript submitted for publication.

Herrmann, Christian/Gerlach, Erin (in Überarb.): The influence of perceived teaching quality on student interest and willingness to put effort in physical education. Manuscript submitted for publication ( $2^{\text {nd }}$ Version).

Kunter, Mareike/Trautwein, Ulrich (2013): Psychologie des Unterrichts. Paderborn: Schöningh.

Linka, Tim/Gerlach, Erin (2019): Klassenmanagement im Sportunterricht - Ergebnisse zur Evaluation der ersten Phase eines videogestützten Seminarkonzepts für angehende Lehrkräfte. In: Hartmann, Meike/Laging, Ralf/Scheinert, Christian (Hrsg.): Professionalisierung in der Sportlehrer*innenbildung. Baltmannsweiler: Schneider, S. 95-107.

Mess, Filip/Ossig, Maren/Woll, Alexander (2014): Bewegte Pausengestaltung. Übungs-/Spielesammlung für Lehrer und Schüler. Schorndorf: Hofmann.

Niederkofler, Benjamin/Amesberger, Günther (2016): Kognitive Handlungsrepräsentationen als Strukturgrundlage zur Definition von kognitiver Aktivierung im Sportunterricht. In: Sportwissenschaft, 46, S. $188-200$.

Sibley, Benjamin A./Etnier, Jennifer (2003): The Relationship between Physical Activity and Cognition in Children: A Meta-Analysis. In: Pediatric Exercise Science, 15, pp. 243-256.

Schmidt, Mirko/Egger, Fabienne/Benzing, Valentin/Jäger, Katja/Conzelmann, Achim/Roebers, Claudia M./Pesce, Caterina (2017): Disentangling the relationship between children's motor ability, executive function and academic achievement. In: PloS one 12, 8, e0182845. DOI: 10.1371/journ al.pone.0182845.

Seiler, Sara (2019): Lernleistungen im Sportunterricht. Wiesbaden: Springer VS.

Tomporowski, Phillip/McCullick, Bryan/Pendleton, Daniel M./Pesce, Caterina (2015): Exercise and children's cognition: The role of exercise characteristics and a place for metacognition. In: Journal of Sport and Health Science, 4, 1, pp. 47-55.

Vogler, Jolanda/Messmer, Roland/Wibowo, Jonas/Heemsoth, Tim/Meier, Stefan (2017): Drei Zugänge zur Modellierung fachdidaktischen Wissens von Sportlehrpersonen. In: Balz, Eckart/Kuhlmann, Detlef (Hrsg.): Sportwissenschaft in pädagogischem Interesse. Hamburg: Czwalina, S. 47-55.

Wibowo, Jonas/Krieger, Claus/Frederik Buekers (Hrsg.) (2019). Aktivierung im Sportunterricht. Hamburg: Universität. https://www.oa.uni-hamburg.de/aktivierung-im-sportunterricht [Zugriff: 10.01.2019]

Zimmer, Renate (2009): Toben macht schlau! Bewegung statt Verkopfung. Freiburg: Herder. 